\title{
FAR-IR GALAXY COUNTS EXPECTED IN THE IRIS SURVEY
}

T.T. TAKEUCHI, H. HIRASHITA, T. G. HATTORI AND K. OHTA Dept. of Astronomy, Kyoto Univ.

Kitashirakawa Oiwake-cho, Sakyo-ku, Kyoto, 606-01, Japan

AND

H. SHIBAI

Division of Particle and Astrophysical Sciences, Nagoya Univ. Furo-cho, Chikusa-ku, Nagoya 464-01, Japan

\section{Introduction}

Infrared Imaging Surveyor (IRIS, officially Astro-F) is a satellite which will be launched in the winter of 2003. The main purpose of the IRIS mission is an all sky survey in the mid- and far-IR with a flux limit much deeper than that of IRAS. In order to examine the performance of the survey and to find a suitable set of bandpasses for tracing galaxy evolution and picking up protogalaxy candidates as effective as possible using IRIS, we estimated the FIR galaxy counts based on a simple model with various sets of cosmological parameters and evolution types.

\section{Method and Conclusion}

We adopted a multicomponent model consisting of cirrus and starburst components for galaxy spectra, and the nearby FIR luminosity function derived from that of IRAS galaxies. We derived the $\log N-\log S$ and $N$ $z$ relations for: 1) no evolution, 2) pure luminosity evolution, and 3) pure density evolution with various sets of cosmological parameters $\left(H, q_{0}\right)$. The results are consistent with those obtained with IRAS, ISO, and COBE. Picking up protogalaxy candidates only by FIR colors is possible, although the contamination of nearby faint objects would be significant. 